\title{
Factores que inciden en el proceso de enseñanza - aprendizaje del español como segunda lengua (L2), segundo grado, escuela "Santo Tomás de Umbra", segundo semestre del año 2007.43
}

Leandro Pekitle Pedro y Hernacio Pedro Maibeth ${ }^{44}$

Eloy Frank Gómez ${ }^{45}$

\section{Resumen}

La temática se fundamenta en los factores que inciden en el proceso de enseñanza - aprendizaje del español como segunda lengua (L2), segundo grado de Educación Primaria, escuela bilingüe en la comunidad: "Santo Tomás de Umbra", segundo semestre del 2007. Asimismo, identifica los factores socioeconómicos que influyen para que la niñez aprenda hablar el español durante su escolaridad. La metodología aplicada fue participativa, activa e interactiva entre los investigadores y los participantes en la investigación (la comunidad) dado el enfoque etnográfico de la investigación, se aplicaron instrumentos como guías de entrevistas semi - estructuradas y grupos focales para madres y padres de familia, estudiantes, docentes, directores de la comunidad.

Los resultados reflejan diversas situaciones que dificultan que la niñez aprenda a hablar el español como segunda lengua para la socialización y necesidades de comunicación con otras personas, destacando el factor pedagógico, la ausencia de capacitaciones, el desconocimiento y aplicación de metodologías pertinentes para la enseñanza de segunda lengua por parte de la maestra. Entre las conclusiones es que uno de los mayores problemas que incide de forma negativa en el aprendizaje del español, es la no aplicación de una metodología pertinente y el desconocimiento de las habilidades lingüísticas para que la niñez pueda ampliar su repertorio verbal para diversos fines. Así como la poca práctica del español por parte de la niñez en la comunidad. Por lo cual se recomienda a las instancias correspondientes como el MINED- PEBI, tomar cartas en el asunto para brindar mayor atención a los docentes de la comunidad, en materia de capacitación y especialmente en aplicación de metodologías para enseñanza del español y conocimientos básicos sobre lingüística.

\footnotetext{
$\overline{43}$ Estudio realizado con el financiamiento del proyecto PATHWAY II "Support to Graduation Research Projects of Indigenous and Afro Descendant Students in URACCAN”, de la Fundación FORD.

44 Egresados de la Carrera de Licenciatura en Educación Intercultural Bilingüe de URACCAN Recinto las Minas.

45 Coordinador de IPILC, URACCAN Recinto Las Minas. Tutor de la investigación.
} 
Palabras claves: Proceso enseñanza - aprendizaje, escuela, lengua, comunicación, metodología, capacitación, práctica pedagógica.

\section{Introducción}

Santo Thomas de Umbra (Sutak Was) es la única comunidad mayangna de hablantes en lengua panamahka, ubicada en la zona de la cuenca media del río Coco o Wangki Awala. A pesar de encontrarse rodeada de comunidades miskitus, y hasta el día de hoy que han resistido ante las presiones culturales y lingüísticas por grupos mayoritarios, manteniendo su identidad y su lengua, como uno de los tesoros más sagrados de la herencia de sus ancestros.

En esta comunidad histórica, las madres y padres de familia afrontan la preocupación porque sus hijos tienen que migrar hacia otros municipios en busca de superación; no obstante, el problema más sentido es no dominar el español, es por eso que nuestra investigación apunta a encontrar los factores que afectan el aprendizaje de la segunda lengua y de esa manera contribuir a buscar alternativas mediante propuestas educativas. La idea obedece a situaciones educativas de carácter pedagógico y tiene sus antecedentes desde el inicio del Programa de Educación Bilingüe Intercultural -PEBIen el año 1984, para las comunidades miskitus y en el contexto de las comunidades mayangnas. Este programa inicia a partir del año 1985 como proyecto piloto en el asentamiento Españolina, con niñas y niños mayangnas refugiados de guerra, de la zona de río Coco que fueron trasladados a estas zonas, que luego se conoció como proyecto "Tasba Pri”.

Esta investigación se enmarca en el análisis de una de las deficiencias pedagógicas más agravante que ha enfrentado el Programa de Educación Bilingüe Intercultural en las escuelas sumu mayangna, como es la enseñanza aprendizaje del español como segunda lengua en la niñez del nivel de Educación Primaria, en la comunidad de "Santo Tomas Umbra" en la zona del río Coco. Obviamente los objetivos de la Educación Intercultural Bilingüe son claros en sus propósitos, donde se menciona que la niñez una vez finalizada su primaria, estará apta y con un buen nivel de competencia comunicativa del español dentro y fuera de la comunidad; Sin embargo, hasta la fecha hablamos de 23 años de educación bilingüe y los cambios en este aspecto no han sido significativos. En todo caso, se parte de la premisa que todo esfuerzo de la Educación Intercultural Bilingüe (EIB) debe encaminarse a lograr un cambio cualitativo tanto en el docente en cuanto la enseñanza y en el caso de los estudiantes indígenas el aprendizaje de la lengua. Todos con fines comunicativos en los diversos contextos de la 
sociedad, lo que se logrará mediante una buena formación y aplicación metodológica para la niñez.

Con esta investigación, se trata de promover un espacio de reflexión con relación al proceso de enseñanza y aprendizaje del español como segundo lengua en la escuela primaria, Profundizar y abordar los factores pedagógicos que afectan la adquisición de la lengua, los factores lingüísticos y socioeconómicos. La niñez mayangna al egresar de la Escuela Primaria Bilingüe no se comunica de manera fluida, ni hace el intento de hablar la lengua del idioma español. ¿A qué se debe? Es lo que queremos llegar a conocer por medio de nuestra investigación de carácter pedagógico y sociolingüístico.

\section{Revisión de literatura}

La Educación Intercultural Bilingüe (EIB) se concibe como una propuesta educativa innovadora y diferenciada de los demás modelos educativos, que propicia aprendizajes significativos basados en la pertinencia de los pueblos indígenas y étnicos. Una de los aspectos que constituye el centro de desarrollo para una adecuada Educación Intercultural Bilingüe, (EIB) es la capacitación y formación de los docentes, talleres específicos en el tratamientos de temas innovadores que respondan a las necesidades y expectativas del PEBI, así como se ha venido trabajando con las escuelas de aplicación pedagógicas del FOREIBCA, SAHWANG y BASE II en pro del desarrollo curricular para reforzar el sistema de seguimiento, monitoreo y evaluación de los docentes (Dixon, 2002, p. 27).

El bilingüismo constituye el foco principal de atención, en relación a las diferencias étnico-culturales. Con esta política se favorece el modo de transición que alfabetiza y enseña en lengua materna como un paso intermedio para el posterior empleo del español. Aun cuando se produce un reconocimiento de las lenguas, ello no significa una valoración de las culturas originarias. Persiste el enfoque asimilasionista, pues finalmente lo que interesa es que el indígena se integre a la cultura dominante, valorada por su prestigio, pujanza y estatus (Venezia, 2003, p.87).

Tal a como lo plantea López (2003): “La enseñanza del español como segunda lengua L2, suele tratarse de un curso de duración variable, de carácter transitorio con un fin práctico e inmediato, facilitar a los nuevos estudiantes el acceso a un sistema académico que se desarrolle enteramente en una segunda lengua (L2), es decir, que utiliza una única lengua para la relación entre sus miembros y para la transmisión del currículo (López. 2003, p. 243). 


\section{EDUCACIÓN}

El idioma español constituye un vehículo a través del cual las personas de origen sumu - mayangna pueden alcanzar mayores calidades educativas y mejores oportunidades de trabajo en las ciudades, también en las relaciones sociales (Rizo 2003, p.76).

La formación del docente para la Educación Intercultural Bilingüe, es un proceso que requiere de una serie de competencias pedagógicas y lingüísticas para la docencia, para promover los valores de las culturas dentro y fuera de la escuela. Es por eso que la práctica pedagógica debe de ser orientada hacia el aprendizaje significativo (Venezia, 2003, p. 98).

Aunque en algunos casos la socialización del español está basado en diversos intereses de la familia y de la misma sociedad. "Algunos padres y madres de familia jóvenes tienen experiencias de la migración hacia las ciudades urbanas y por ende, socializan a sus hijas e hijos en la adquisición del español como primera lengua, considerando a esta como un medio fundamental para alcanzar un mayor nivel de educación y mejores oportunidades de trabajo en la ciudad. Mientras que los que socializan a sus hijas e hijos en la adquisición de la lengua indígena, lo perciben a esta lengua, como un obstáculo para transitar a otros espacios sociales (De la Torre, Luís 1997, p. 58).

La Educación Intercultural Bilingüe es un proceso planificado en el aula, donde se enseña la lengua materna de la niñez así como una segunda lengua, esto significa que los docentes realizan actividades curriculares, incluyendo la evaluación en las dos lenguas, teniendo como referencia un plan específico para su desarrollo (Venezia,2003, p. 36).

La enseñanza del español como segunda lengua debe realizarse mediante las artes del lenguaje, el programa debe ser integral; es decir, primero, enseñar a escuchar, luego a hablar y posteriormente a leer y escribir, cada una de las artes del lenguaje merece un tratamiento didáctico específico (Venezia, 2003, p.56).

El primer principio importante de la estrategia oral se fundamenta en la aseveración hecha anteriormente: "Hablar antes de leer", la gente a veces mal entiende el énfasis central de la estrategia oral considerando como una prohibición del uso de la lectura y de la escritura durante las primeras semanas o meses de estudio de un idioma. Pero, la estrategia oral no implica tal prohibición, la escritura y los materiales impresos pueden ser muy útiles en la estrategia oral (Muñoz, 2003, p. 19).

Los materiales didácticos, actualmente en uso en las escuelas bilingües no contribuyen a dinamizar el desarrollo curricular y no facilitan el trabajo grupal, son utilizados 
fundamentalmente para actividades repetitivas y de copiados (IPILC-URACCAN, DEC, 2003, p. 39).

Los tipos de enseñanza de segundas lenguas en el contexto escolar pueden reducirse a dos tipos esenciales según la presencia de la L2 en el currículo. Por otra parte, tenemos la enseñanza bilingüe o multilingüe, cuyo objetivo principal es posibilitar el dominio de dos o más lenguas a las que el escolar no tiene acceso en su medio social y familiar. Las nuevas lenguas objeto de aprendizaje tienen un carácter instrumental y son vehículos de las actividades de enseñanza - aprendizaje de cualquier contenido en cualquier área educativa, no sólo en el área de lenguaje. Se excluyen de este concepto, por lo tanto, situaciones en las que se enseña la L1 o L2 sólo como materia (Freeland, 2003, p.49).

Estas coincidencias han dado lugar a que investigaciones sobre enseñanza bilingüe hayan sido aplicadas a muchos aspectos de la enseñanza de L2. Empero, los rasgos sociolingüísticos de los alumnos a los que van dirigidas, así como los fines y la organización de los programas, hacen que prefiramos con muchos otros mantener la distinción y ver en ellas dos campos de enseñanza diferenciada (Bolívar Chiriboga, 2001. p. 27).

El acceso a la lengua de instrucción en este caso el español, se convierte no sólo en la lengua de comunicación, sino también en lengua de aprendizaje, fundamental en el ámbito escolar y necesario para la inserción en el mundo académico o laboral. El desarrollo de la persona, aprendiendo a ser y a convivir en una sociedad plurilingüe y pluricultural, al amparo de unas normas y de unos valores universales, y al servicio de la adquisición de actitudes de cooperación y de participación en igualdad de condiciones (García Armendáriz. 2003, p.14).

Otro escollo en la adaptación del estudiante a su nuevo ámbito escolar tiene que ver con la cultura lingüística que trae consigo y que puede ser diferente de la que encuentra en el nuevo entorno (Ambadiang, 2003, p. 99).

Savinnon (1983) ha clasificado que la comunicación como un concepto dinámico que depende de la negociación del significado entre varias personas, se vuelve así aludir a la necesidad de ofrecer en el aula tareas que promuevan la interacción, ya que se considera la competencia comunicativa como un elemento de base interpersonal.

Aunque exista una multiplicidad de variables que el docente de una segunda lengua ha de atender para el desarrollo de la competencia comunicativa oral, tampoco se puede olvidar que el alumno de una L2 ya viene equipado con ciertas capacidades adquiridas 


\section{EDUCACIÓN}

en la L1. El conocer cuáles son y el cómo ayudar al aprendiz para que transfiera dichas habilidades puede ser de una inestimable ayuda para el desarrollo de una enseñanza eficaz de la competencia comunicativa oral, según Bachean y Palmer (1996, p. 70).

El uso de las diferentes lenguas en la práctica pedagógica está vinculado a los objetivos comunicativos y a las capacidades lingüísticas del docente y estudiante. En contexto monolingüe la introducción de la segunda lengua comenzará con el desarrollo de la expresión oral, mientras que la niñez es introducida a la lecto - escritura en su lengua materna. En segundo grado, mientras se afianzan las habilidades comunicativas orales y la lecto - escritura en la L1 se comenzará la transferencia de habilidades al español. (Muñoz 1997, p. 57).

Todas las lenguas hacen uso de un sistema de sonidos limitados y hay sonidos que se encuentran en todas las lenguas como: a, i, u, p. t. k, pero no siempre estos sonidos son iguales, la lengua puede tener un sonido propio y que no comparte con otras lenguas (Venezia, 2003, p.153).

En las escuelas interculturales bilingües también se deberá considerar la necesidad de contar con materiales para auto aprendizaje, necesarios para las escuelas multigrados y bibliotecas escolares. Es importante comenzar una reflexión sobre tecnologías educativas, para elaborar un programa de difusión y aprovechamiento de dichas tecnologías (Diagnóstico Educativo Comunitario DEC, 2003, p. 40).

En las comunidades alejadas del río arriba de Waspam río Coco, las condiciones son totalmente diferentes. Es importante mencionar que las zonas más pobres y lejanas, donde las condiciones de infraestructura son deplorables, en los casos donde existen escuelas. En muchos casos se prestan las iglesias y casas particulares para poder desarrollar las clases. La infraestructura escolar es deficiente, en algunas comunidades hacen falta escuelas y en otros casos hacen falta más aulas y plazas para docentes, incluso edificios escolares recién construidas no presentan las condiciones adecuadas para la práctica pedagógica y en muchos casos son inadecuados para atender a toda la población estudiantil de la comunidad o barrio, muchas escuelas están en mal estado, descuidadas y sucias (Dixon, 2002, p. 27).

El calendario escolar impide el acceso y permanencia de la niñez en la escuela, porque no toma en cuenta las épocas de siembra, cosecha y pesca. El sistema escolar no funciona con un calendario adecuado a los ciclos de producción y a las necesidades de las comunidades. Esta limitante es importante para el ejercicio del derecho a la educación, porque en todas las comunidades participantes en el $\mathrm{DEC}^{46}$, las actividades económi-

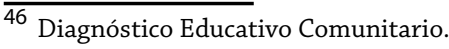


cas principales son la agricultura y la pesca, los habitantes no tienen otras formas de ingreso y aseguramiento de la alimentación y satisfacción de sus necesidades básicas de sobrevivencia de las familias. En tiempo de siembra, pesca y cosecha las niñas y niños acompañan a la familia en el trabajo, y esto generalmente tiene consecuencias graves para la reinserción y promoción de un grado a otro (Diagnóstico Educativo comunitario -DEC-, 2003, p. 56).

También Confiant (2007) expresa que el MED, ha enfrentado numerosas y serias dificultades en sus esfuerzos por materializar y desarrollar el programa de educación bilingüe intercultural, por las condiciones mismas de la región; el clima, su topografía, la carencia de vías de acceso terrestre y acuático, el aislamiento geográfico y cultural de las comunidades.

El fracaso escolar, es un término subjetivo que viene determinado por circunstancias muy cambiantes, históricas, económicas y socioculturales que rodean al acto educativo. Por ello es un concepto que provoca bastante discusión, porque por un lado transmite la idea del alumno fracasado que no ha progresado prácticamente nada por la situación de la inasistencia escolar (Dorn, 2002, p.230).

El Instituto y Desarrollo Pedagógico (IDEP) de la Secretaría de Educación de Bogotá manifiesta su preocupación por el crecimiento de las dificultades de aprendizaje como consecuencia de las enfermedades que afectan a los educandos por lo que se requiere de la utilización de pedagogías flexibles y acorde con las circunstancias de la salud de los niños. Dificultades de aprendizaje disponible en: http://www.universia.net.co/ noticias/noticia-del-dia/la-dificultad-de-aprender.htm/.

En el Ministerio de Educación el registro de archivo escolar comprende normativas de registro de calificaciones, registro de estadística, donde se lleva la matricula inicial, actual y final de los estudiantes, registro de notas acumuladas por parte de los estudiantes. En cada uno de los centros escolares deberá de existir el libro de acta, donde se registran todos los estudiantes promovidos y aplazados, fecha de reparación y promovidos, demás debe de existir el cuadro de notas por sección, por cada alumno, por parciales y semestrales, el registro de la escuela es imprescindible para conservar todo el historial del centro (Entrevista Godoy Alfaro 22 mayo 2008).

Materiales y métodos: El estudio se desarrolló en la comunidad de "Santo Tomás de Umbra” del municipio de Waspam río Coco (Wangki Awala) Departamento de la Región Autónoma del Atlántico Norte RAAN, es cualitativo con enfoque etnográfico. La unidad de análisis fueron la comunidad estudiantil, padres y madres de familia, director, docente de segundo grado de la Escuela Bilingüe "Chepita Toledo". 
Las técnicas utilizadas durante la investigación fueron: Entrevistas semi-estructuradas, grupos focales, observación participante. Los instrumentos que se utilizaron fueron la guía de entrevista, guía para grupo focal y la guía de observación participante, asimismo el diario de campo.

\section{Resultados y discusión}

Factores pedagógicos que inciden en la enseñanza aprendizaje del español como segunda lengua.

Capacitación: La capacitación es el eje para que el docente tenga todas las herramientas que le permita desarrollar una mejor clase, utilizando prácticas pedagógicas que estén a la altura de las exigencia de las metodologías educativas. En el contexto de las Escuelas Bilingües se requiere de constante actualización a raíz del tratamiento especial que se debe promover con las lenguas y la cultura. Antes bien, la realidad que encontramos en la comunidad de "Santo Tomas Umbra" es muy preocupante, los docente están realizando lo imposible por poder llevar la enseñanza, por el abandono y la poca preparación que han tenido.

Al respecto Dixon ( 2002) hace referencia a la importancia que se le debe proporcionar a las capacitaciones, cuando afirma: "Uno de los aspectos que constituye el centro de desarrollo para una buena Educación Intercultural Bilingüe (EIB) es la capacitación y formación de los docentes de las escuelas, talleres específicos en tratamientos de temas innovativos, para que respondan a las necesidades y expectativas del PEBI, así como se ha venido trabajando con las escuelas de aplicación pedagógicas del FOREIBCA, SAHWANG y BASE II en pro del desarrollo curricular. Empero, en el caso de la comunidad de "Santo Tomas" no estuvo atendida por ninguno de los proyectos) que se desarrollaron en la R.A.A.N. tal a como hace mención (Dixon 2002). Las razones se desconocen, aunque la maestra de la escuela dice que es posible que sea por la distancia en que se encuentra la comunidad con relación a la sede del PEBI mayangna en Las Minas. En otro orden, la situación geográfica no pudo constituirse en factor para no tomar en cuenta a los docentes, porque, uno de los proyectos como BASE II estuvo atendiendo la comunidad de "San Carlos", que está a 15 minutos de "Santo Tomás", aunque el director plantea otras razones de discriminación por parte de las autoridades educativas.

La realidad educativa por la que atraviesa la maestra de la escuela "Santo Tomás" difiere con relación al planteamiento de Venecia (2003) cuando dice: "La formación del docente para la educación intercultural bilingüe, es un proceso que requiere entre 
otras cosas, la adquisición y la práctica de una serie de competencias pedagógicas y lingüísticas para la docencia, la interculturalidad y el plurilingüismo, para promover los valores de las culturas dentro y fuera de la escuela. Es por eso que la práctica pedagógica debe de ir orientada hacia el aprendizaje significativo". No obstante, la maestra carece de tales conocimientos por no tener la oportunidad de capacitarse en el Programa de Educación Bilingüe Intercultural -PEBI-. Los padres y madres de familia consideran que si la maestra después de haber participado en un taller de capacitación no aplica ese conocimiento en la escuela, los líderes de esta comunidad tendrían suficiente elementos para reclamarle a la maestra por el aprendizaje de la niñez; antes bien, como no ha recibido ninguna capacitación, ellos reconocen el esfuerzo que hace la maestra por enseñarles algo a sus hijas e hijos. Ante las diversas manifestaciones encontradas en el aula de clase, existen opiniones adversas con respecto a lo que destaca el director del centro y la misma maestra, destacando acciones de muy poca preocupación por los maestros en la comunidad, "porque muchos se preparan y no transmiten ese nuevo conocimiento". El juez de la comunidad fue uno de los que considera que la maestra no está cumpliendo con su responsabilidad.

Para la mayoría de los comunitarios, el tema de las capacitaciones de los docentes y la situación de aprendizaje de los estudiante, lo aprecian como responsabilidad de la institución rectora del MINED, sobre todo el delegado de esa institución; a la misma vez sugieren que antes de iniciar un año lectivo se capacite a los docentes para que estos puedan a enseñarles bien a la niñez. Principalmente en esos años, porque los técnicos o materiales no están actualizados y los docentes sin capacitarse no podrán lograr un avance significativo, aunque los docentes tengan miles de experiencias y conocimientos, no es lo suficiente para enseñarles pertinentemente conforme a la realidad.

De la Torre (1997) refiere que "algunos casos de socialización del español está basado en diversos intereses de la familia y la misma sociedad, las migraciones hacia las ciudades urbanas por lo tanto consideran de mucha importancia la adquisición del español ya que les sirve como un medio fundamental para alcanzar un mejor nivel de educación y mejores oportunidades de trabajo en la ciudad".

Medios y recursos: Durante la observación de clase, se detectó la ausencia de suficientes medios y recursos para el desempeño de la práctica pedagógica; sin embargo, la maestra con su experiencia elabora sus materiales de enseñanza, ya sean láminas en papelógrafos o cartulina, pero la escuela no cuenta con estos recursos por lo tanto tiene que comprarlos o no consigue materiales concretos que sean del medio natural y que el niño los conozca. En tales circunstancias, el proceso de enseñanza aprendizaje de los niños se vuelve más monótono, pierde el dinamismo y se le hace más difícil la 


\section{EDUCACIÓN}

comprensión de los significados de las palabras que enseña la maestra en español, la escuela no cuenta con recursos como cartulinas, papelógrafos, una buena pizarra, la maestra escribe en unas pedazos de tabla que fue construida por los padres de familia. Esta situación tiene similar relación con lo que describe un estudio realizado por el URACCAN - IPILC, sobre la situación de la educación en las escuelas bilingües, cuando plantean que las Escuelas Interculturales Bilingües deberán considerar la necesidad de tener materiales para el aprendizaje, necesarios para las escuelas multigrados y bibliotecas escolares.

Es importante comenzar una reflexión sobre tecnologías educativas, para elaborar un programa de difusión y aprovechamiento de dichas tecnologías. (Diagnóstico Educativo comunitario DEC, 2003, p. 40). La necesidad de disponer con otros recursos para la enseñanza, así como nuevas tecnologías educativas, en el caso de esta comunidad de "Santo Tomás", está muy lejos de adquirir dichos recursos y materiales, cuando no son atendidos por ninguna de las instancias educativas como son la delegación del MINED del municipio de Waspam y la dirección del Programa de Educación Bilingüe Intercultural mayangna en Rosita, de esta manera se coincide con los planteamientos de muchas madres y padres de familia de la comunidad. Es evidente la falta de apoyo que tiene esta comunidad en materia educativa; empero, el director del centro considera que la ausencia de dichos recursos no impide que la maestra pueda poner en práctica la experiencia de su trayectoria como educadora para poder enseñarles a la niñez.

Condiciones del aula: Uno de los elementos que contribuye al aprendizaje de los educandos, es el ambiente escolar en la que incluye las condiciones del centro escolar y en específico el aula de clase. Con relación a las condición del aula de 2do grado, se pudo constatar que la infraestructura de la escuela en su totalidad está muy deteriorada, la madera está podrida, el zinc fue despegado por los efectos del Huracán Félix, y los comunitarios volvieron a poner el zinc, aunque cada vez que llueve se moja toda. Se pudo observar que no hay suficiente muebles, muchas niñas y niños están sentado en el piso, el aula como tal no está forrada, solamente clavada con unos palos para evitar la entrada de personas ajenas. Dixon puntualiza: "La infraestructura escolar es deficiente, en algunas comunidades hacen falta escuelas y en otros casos hacen falta más aulas y plazas para docentes, incluso edificios escolares recién construidos no presentan las condiciones adecuadas para la práctica pedagógica, y en muchos casos son inadecuados para atender a toda la población estudiantil de la comunidad o barrio, muchas escuelas están en mal estado, descuidadas y sucias (2002, p. 27).

La situación del aula, no presta las condiciones pedagógicas mínimas, no tiene espacio para ubicar los rincones de aprendizaje para cada materia o área, ya que los rincones de aprendizaje son recursos auxiliares para que las niñas y niños tengan acceso de 
consultar materiales para sus investigaciones. Al consultar a la maestra con relación a como hacía para trabajar cuando llueve ella manifestó: "La escuela está en pésimas condición, casi hasta por caerse y esto nos desmoraliza en el desempeño del trabajo, cuando uno trabaja dentro del centro nuevo, bonito y el aula tiene buena condición a los niños también les llama la atención para permanecer en el aula, no quieren faltar ni un día de clase (Entrevista Modestina Charly, Mayo 2008.). La maestra explicó que por la condición del aula, no se pueden dejar los trabajos que los alumnos efectúan, porque al llegar al día siguiente no los encontraban, porque cualquiera entra y se lleva las cosas a su casa. Hay inseguridad, por lo que decía que es necesario que algún organismo o institución, piense por lo menos en rehabilitar el centro.

Registro de archivos: Se presentan muchas irregularidades en la escuela, no existe nada del archivo escolar que debería tener la dirección de la escuela, todo lo que tiene que ver con los expediente del docente, historial académico de los estudiantes por grado, hay un vacío como tal. Se pudo constatar que no existe ningún libro de acta para llevar el control de todo las reuniones que se hacen con la comunidad, mucho menos un archivo que se guarde la historia de la comunidad, las promociones y otros elementos importantes en el funcionamiento de la escuela. Cuando se les preguntó a las madres de familia, una de ella se refirió así: "Si una maestra no llega con el plan diario ante un grupo de niños, para qué sirve la enseñanza, debe de planificar bien antes de presentarse ante los niños y el proceso de enseñanza será buena y la meta propuesta en su plan podrá lograrse sin tener problemas (Entrevista Elida Charly López: Abril 2008).

El director del centro explicaba que el deber de cada docente es llevar el control diario de los estudiantes, cada uno debe tener sus propios archivos, para saber quien se ausenta cada día, para así rendir el informe estadístico mensual. Cada docente lleva una orden de calificación para estar muy claro cuáles de los niños son eficientes y cuáles no. Aunque existe mucha diferencia con lo que dice el director del centro, ya que durante el trabajo con el grupo focal, los padres de familia expresaron que desde hace más de tres años a ellos no les entregan el boletín de sus hijos, no saben si realmente sus hijos aprobaron el año, porque no tienen cómo ver las calificaciones, es por eso que consideran que no existe nada de los archivos en la escuela, en la dirección no existe nada ni un fólder con algún papel o una acta de reunión del centro. De igual manera estos planteamientos se diferencian con lo que plantea (Godoy 2008) que todos los centros deberán de contar con sus registros de archivo ya que es imprescindible para conservar el historial de la escuela, eso implica, registro de estadística, registro de calificaciones de todos los estudiantes promovidos y aplazados, fecha de reparación, el libro de acta donde se lleva, por parciales y semestrales, El registro de la escuela es imprescindible para conservar todo el historial del centro (Godoy Alfaro 2008). 
Supervisión: Durante las conversaciones con los docentes manifestaban que desde hace muchos años los técnicos del Ministerio de Educación no visitan este centro, no supervisan a los maestros cuando están desarrollando las clase, el delegado del MINED llegó una sola vez a la comunidad, pero sólo durante la campaña electoral; después solamente llegaban hasta la comunidad de San Carlos y se regresaban a Waspam. Los maestros no tienen ningún apoyo de nadie, nadie les facilita seguimiento ni orientaciones de cómo trabajar mejor en la escuela, prácticamente el centro se encuentra aislado. De hecho para que la educación marche bien en la escuela es preciso fortalecer el seguimiento y acompañamiento a las escuelas, tal a como refiere Rosales (2001) cuando identifica que para lograr las tareas de los docentes indígenas y cumplir con su rol de educador, y que esto conlleve a resolver las situaciones problemáticas de carácter pedagógico, los maestros necesitan ser apoyados teórica y metodológicamente mediante acompañamiento en sus centros escolares.

Cuando se solicitó alguna copia de observación de clase o de algún informe que haya dejado algún técnico, no se encontró absolutamente nada, ya que no existe ningún tipo de documento de ese tipo. En los talleres con grupos focales de padres de familia decían que los maestros andan a la libre, cuando querían impartían clases y si no querían no; cuando los padres les reclamaban a los maestros, estos contestaban diciendo que a ellos nadie les tiene que decir nada, por eso ellos son preparados, situación que contradice con el compromiso de los estudiantes que vienen a prepararse en las universidades, donde una vez que finalizan sus estudios deberán regresar a sus comunidades a servir a su pueblo, y esto implica trabajar por mejorar la situación de la educación con miras a la calidad. Aunque Confiant (2007) considera que existen muchos factores que influyen para que las instancias educativas no les proporcionen seguimiento y supervisión a muchos centros del país, cuando expresa que el MECD, ha enfrentado numerosas y serias dificultades en sus esfuerzos por materializar y desarrollar el Programa de Educación Bilingüe Intercultural, por las condiciones mismas de la región; el clima, su topografía, la carencia de vías de acceso terrestre y acuático, el aislamiento geográfico y cultural de las comunidades. Por otro lado, se suma la ausencia de personal técnico metodológico mayangna en la delegación del MINED de Waspam, para que pueda atender la comunidad de "Santo Tomas" en la modalidad bilingüe.

Asistencia de estudiantes: En la mayoría de las madres y padres de familia, consultados sobre la situación de la asistencia, en la mayoría coincidían en las respuestas al decir que uno de los problemas más sentido en la escuela de "Santo Tomas" son los casos de inasistencia en el periodo de siembra, pesca y cosecha, porque las niñas y niños acompañan a la familia en el trabajo, y esto generalmente tiene consecuencias graves para la reinserción al aula, así como la promoción de un grado a otro. Ante tal 
caso, esta situación encontrada en la escuela de "Santo Tomás" coincide con lo que expone Dorn (2002) cuando se refiere que: "El fracaso escolar, es un término subjetivo que viene determinado por circunstancias muy cambiantes, históricas, económicas y socioculturales que rodean al acto educativo. Por ello es un concepto que provoca bastante discusión, ya que por un lado transmite la idea del alumno fracasado que no ha progresado prácticamente nada por la situación de la inasistencia escolar".

\section{Factores lingüísticos que inciden en la enseñanza aprendizaje de español como segunda lengua}

Fonemas: Durante las observaciones de clase se constató que existen algunos fonemas donde hay más problemas de pronunciación estos son: f/por la p/, g/ por la k/, la otra situación se produce en la escritura donde utilizan los grafemas del idioma mayangna cuando escriben palabras en español, es allí donde la maestra considera que se le complica mucho para ayudar a los niños, porque no ha recibido ningún curso sobre lingüística mayangna.

Los fonemas de L2 no son iguales con los de L1, tienen formas diferentes en algunos ciertamente son iguales como por ejemplo: a, i, u, y, t, p, m, r, b, d, n, s, w, g, son los mismos que se utilizan en los dos idiomas. Sin embargo, a veces los niños presentan dificultades en la pronunciación, ya que para algunos se les dificulta identificar los fonemas de cada idioma, por eso a veces se confunden (Entrevista, Modestina Charly 2008). Lo que plantea la maestra y lo que se pudo constatar durante a observación de clase tiene relación con lo que plantea Venecia (2003) cuando refiere que todas las lenguas hacen uso de un sistema de sonidos limitados y hay sonidos que se encuentran en todas las lenguas como: a, i, u, p. t. k, pero no siempre estos sonidos son iguales, la lengua puede tener un sonido propio y que no comparte con otras lenguas (Venezia, 2003, p.153).

La maestra manifestó que la niñez de segundo grado conocen los fonemas del lenguaje español; pero no todos, ya que el tiempo de permanencia en la escuela no es lo suficiente para poder asimilar. También las madres y padres tienen que poner de su parte, es por eso que muchos niños regresan a sus casas a como llegaron, además que no practican lo poco del español que se les enseña en la escuela. Aunque esta situación de identificación de los fonemas y el mismo aprendizaje del español no solamente es porque los padres y los mismos niños no ponen atención, sino porque intervienen otros factores pedagógicos que les dificulta poder comprender los fonemas en ambas lenguas, teniendo mucha relación con lo que plantea De la Torre (1997) que el proceso de adquisición lingüística se relaciona con cuestiones tales como la personalidad, la inteligencia o la edad de los estudiantes, siendo esto una situación real en muchas 
escuelas bilingües rurales, donde en el segundo grado existen estudiantes con edades de 13 a 14 años, por cierto esto marca la diferencia en cuanto el ritmo de aprendizaje, así también sigue remarcando De la Torre, que a esa situación también se suma los factores psicosociales.

A diferencia de lo que se ha observado, también se producen logros sustanciales como los que refiere la maestra de segundo grado: "Los niños conocen los fonemas durante los tres primeros meses, pero conocen más los fonemas de la lengua materna, a la par, aprenden los fonemas de la L2, pero no de manera rápida, algunos niños que ya pueden leer, aprenden más rápido porque saben desenvolverse y en el período de clase practican lo que han aprendido, a través de expresiones cortas." (Entrevista a docente de 2do grado, Mayo 2008). Por lo tanto las aseveraciones de Serra y Vila (2000) tiene mucha relación con lo que explica la maestra al decir, las variedades de un modelo de enseñanza según los fines educativos y objetivos lingüísticos que se pretendan alcanzar en el aula de clase, se relaciona con la lengua que domina el niño y la utilizada en la enseñanza.

Estrategias de enseñanza - aprendizaje: En la Educación Intercultural Bilingüe, se han implementado diversas estrategias de enseñanza del español como segunda lengua, uno de ellos es el método global silábico el cual es el que se está aplicando en las aulas bilingües; empero, en la escuela de "Santo Tomás", no se observó la aplicación de este método para enseñar el español, ya que la maestra de manera improvisada hace el esfuerzo de poder enseñarles algo de la lengua en español sin aplicar ninguna técnica. Situación que no se relaciona con lo refleja el informe del Diagnóstico Educativo Comunitario presentado por el URACCAN - IPILC (2003) al referirse que los métodos de enseñanza de la L2 se conocen como el método global silábico en el que se hace uso de técnicas de audición y reproducción, diálogo docente - grupo, diálogo entre estudiantes, el niño comprende lo que escucha. La transferencia de las habilidades de lectoescritura en L1 a la L2 se iniciará por los sonidos y letras comunes.

La enseñanza del español en el segundo grado, se realiza mediante procesos de repetición de ciertas frases, sin que los niños puedan entender el significado de lo que repetían casi durante toda la clase, existiendo una ausencia práctica de un aprendizaje significativo. Se nota la ausencia de técnicas de enseñanza de las lenguas, no hace uso del orden y las habilidades que integran la lengua, se refiere Venezia (2003), que para la enseñanza de las lenguas, lo primero que se debe trabajar es la escucha, luego hablar y posteriormente leer y escribir, facilitándole a cada uno un tratamiento didáctico específico, situación que no se está trabajando de esta manera, sino a como la maestra lo concibe que debería ser la enseñanza. 
Durante el tratamiento de las lenguas en la escuela bilingüe, se pudo constatar que no se está produciendo un orden lógico de la enseñanza del español como segunda lengua, cuando implica sistematizar la secuencia del las habilidades que los niños traen desde el primer grado, donde todo se trabaja en la fase oral; el otro problema detectado, es que la maestra se encasilla sólo en la parte oral y no en lecto - escritura, donde pueda relacionar lo que dicen los niños y la escritura del mismo. Algunos autores como Muñoz ( 2003) al respecto, sugieren que "se debe abordar las dos cosas, cuando menciona que muchos maestros mal entienden el énfasis central de la estrategia oral, considerando como una prohibición del uso de la lectura y la escritura durante las primeras semanas del estudio de los idiomas".

Para lograr un buen desarrollo de aprendizaje de la L2 en la niñez, es preciso que la maestra planifique las tareas teniendo en cuenta las habilidades comunicativas que deben lograr los niños y niñas, se deben presentar y utilizar aspectos del castellano que se usan para una comunicación más efectiva: solicitar o proporcionar información, pedir un objeto, presentar, sugerir y exponer, pensando en lo más básico que requiere el niño para aprender a hablar la lengua, situación que durante la clase de L2 en la escuela, no se dio. Esto tiene relación con los que Muñoz (2003) explica en cuanto a la estrategia estructural para la enseñanza de idiomas, no se promueve mediante un proceso ordenado de la estructura del idioma, ya que lo normal que se observa en las aulas de clase, se trata de enseñar estos elementos como ítems separados, uno por uno y poco a poco; es decir, no se enseñan cosas sobre los elementos del idioma, sino que la maestra distribuye el tiempo explicando reglas gramaticales.

Como una estrategia pedagógica para el tratamiento del español como segunda lengua, se debe enseñar con las temáticas que el profesor trata de enseñar, esto quiere decir que debe enseñar de acuerdo a la 'situación'; por ejemplo, a través de las situaciones que ocurren en la realidad y que se puede conversar en la clase. En las observaciones de clase la característica que prevaleció fue que proceso de enseñanza estaba dirigido a enseñar reglas y no a aprender el idioma usando recursos expresivos y corporales, los cuales son de mucha importancia durante la enseñanza de las lenguas.

Aunque la maestra de segundo grado, al abordar la situación de la estrategia que ella utiliza para enseñar explicó: "Lo que hago es practicar las expresiones y palpando diciendo, este es una fruta...demostrando las cosas... los niños pueden aprender y entender porque algunos ya traen ciertos conocimientos del español aunque sea mínimo". Ante tal planteamiento de la maestra, Bachean y Palmer (1996) coinciden al respecto cuando opinan: "Aunque exista una multiplicidad de variables que el docente de una segunda lengua ha de atender para el desarrollo de la competencia comunicativa oral, no debe olvidar que el alumno ya viene equipado con elementos 
o palabras en español". En las aulas bilingües, el proceso de enseñanza aprendizaje es variado, es decir el ritmo de los estudiantes se diferencia, por lo mismo que expresa Bachean y palmer (1996), ya que en algunos niños tienen ciertos repertorios cortos del español, porque algunos han salido hacia otras comunidades, esto les ha ayudo a poder entender y familiarizarse con la lengua del español.

En el caso de esta escuela, la maestra explicaba que las primeras frases que aprenden es a saludar, "Buenos días" esta frase se vuelve un lema durante todo el desarrollo de la clase para que no se les olvide. En tales circunstancias, la funcionalidad que debe tener la enseñanza del español como segunda lengua, tiene mucha relación con lo que dice Freeland (2003): "la enseñanza de segundas lenguas en el contexto escolar tiene un objetivo principal, es posibilitar el dominio de la lengua relacionándolo a su medio social y familiar". Por lo tanto no se trata de enseñar la lengua sólo como una materia, sino que la enseñanza esté dirigida para que los niños aprendan a hablar la lengua, situaciones que son muy diferentes.

La maestra explicaba que los padres se preocupan y desesperan, porque piensan que los niños saliendo del segundo grado ya deberían de hablar bien el español; pero, por otro lado ellos no los ayudan a que practiquen en español, no es igual en el caso del miskitu, porque hay niños miskitus y los niños mayangnas se relacionan con ellos para jugar y de esa manera lo aprenden jugando; no obstante, en el caso del español, en la comunidad no hay niños mestizos que sólo hablen español, y esto es otro problema para la niñez ya que no puede poner en práctica lo poquito que se les enseña. Por otro lado, la situación se le complica más a esta niñez ya que si la comunidad fuese una sociedad bilingüe: sumu -español, pues las condiciones estarían dadas. García Armendáriz (2003) dice que el tener accesos a un aprendizaje del español como segunda lengua, no sólo presupone que servirá para la comunicación nada más, sino también, necesario para la inserción en el mundo académico o laboral, pensando que el desarrollo de la persona está ligado a las relaciones con miembros de otras sociedades plurilingüe y pluricultural, en eso radica la importancia de la enseñanza del español para la niñez de las comunidades indígenas.

Otra de las cosas que se constató en el aula de segundo grado, es que hay muchos niños que tienen 13 años de edad y no podían leer, en otros casos leían regularmente; por lo tanto ameritó hacerles una prueba de lectura y esos fueron los resultados que obtuvimos. Esto nos hizo reflexionar alrededor de que el problema de aprendizaje del español es más complejo, ya que esto no concuerda con los estudios de algunos expertos en psicología y lingüistas citado por Skehan, (1998) que plantean que la niñez, antes de leer primero, debe de saber expresarse en la lengua ya que se inicia con la socialización; sin embargo, como se dijo anteriormente, el proceso de socialización 
es posible cuando las condiciones están dadas, es decir cuando hay hablantes de la lengua; pero, en el caso de "Santo Tomas" no hay nadie quien hable el español y esto complica más la situación en la niñez.

Material didáctico: En la dirección de este centro no hay recursos y materiales didácticos para hacer consultas, únicamente lo que la maestra utiliza y se guía, para hacer el plan diario es el libro de texto, no cuenta ni con programa mucho menos guía metodológica para que el docente pueda orientarse mejor en su desempeño escolar. La escuela no tiene ningún apoyo de este tipo, los maestros tienen que proveerse por su propia cuenta de libros, que en algunos casos nada tienen que ver con la educación bilingüe, existiendo así una diferencia con relación a otros centros bilingüe donde muchos proyectos apoyaron con equipamiento de materiales bibliográficos y otros con materiales fungibles.

De acuerdo con el informe presentado por (URACCAN - IPILC: 2003) se refiere que en las Escuelas Interculturales Bilingües también se deberá considerar la necesidad de disponer con materiales para el auto aprendizaje. La maestra siente preocupación con la carencia de materiales; pero, también la necesidad de aprender a utilizar esos materiales a través de la capacitación. Aunque en algunos casos la socialización del español está basado en diversos intereses de la familia y misma sociedad, "Algunos padres y madres de familia jóvenes, tienen experiencias de la migración hacia las ciudades urbanas y por ende, socializan a sus hijas e hijos en la adquisición del castellano como primera lengua, considerando a esta como un medio fundamental para alcanzar un mayor nivel de educación y mejores oportunidades de trabajo en la ciudad. Mientras que los que socializan a sus hijos e hijas en la adquisición de la lengua indígena, perciben a esta lengua como un obstáculo para transitar a otros espacios sociales (De la Torre, Luís 1997, p. 58).

Se presentan otras situaciones con relación a los materiales, la maestra considera que a los niños se les hace difícil aprender con los textos que tienen, porque algunos contenidos están fuera de la realidad de dicha niñez; es decir, para aprender necesita que esos contenidos estén acordes con la realidad y el medio donde se vive, solo así ellas y elos podrán aprender las cosas que conocen, lo que ven y conviven diariamente. Esta es la realidad de la situación de los materiales didácticos en la comunidad de "Santo Thomas", esto no tiene ninguna relación con lo que plantea el (Plan SEAR 2003-2013) el cual expresa que los diseños de los planes y programas interculturales, deben incorporar en los contenidos todo lo relacionado al patrimonio cultural, sistema de valores, tradiciones y medio ambiente, para de esta manera articular el conocimiento propio de su cultura con las prácticas pedagógicas, que potencien sus conocimientos, preparándolos para el contexto actual. 


\section{Factores socioeconómicos que inciden en el aprendizaje de español como segunda lengua}

Ciclo de producción: Uno de los factores que también influyen en que la niñez no pueda tener una permanencia en la escuela y poder mantener una secuencia de los contenidos que desarrolla la maestra, en el caso de la comunidad de "Santo Tomas", tiene que ver mucho con los períodos de siembra y cosecha de los productos. Los meses que más afectan son: marzo, abril, mayo, período de siembra y los meses de agosto, septiembre y octubre que es cuando se da la cosecha de la producción. A la mayoría de los padres a quienes se les consultó sobre cómo la situación de las siembras y cosechas afectaba a los niños en la escuela, la mayoría coincidía al decir que en temporadas de roza y siembra, lo que hacen es que se llevan a los niños más grandes que ya pueden chapear, de igual forma a las niñas; pero, en el último caso, las afectaciones se dan más cuando corresponde el período de siembra ya que los niños que pueden caminar para llegar a la milpa se los llevan para que ayuden a sembrar, es una manera de enseñarles a conservar el conocimiento y la técnica de sembrar, además mencionaron que el otro período que los niños se ven afectados, es durante las cosechas, entre los meses de agosto, septiembre y octubre, aunque este en menos grado. En relación con los períodos de siembra y cosecha en la comunidad de "Santo Tomas", ninguna de las fases del ciclo agrícola coinciden con el período de vacaciones de la escuela, es por eso que se dan muchas afectaciones durante la clase y por ende en el aprendizaje del Español como L2.

De tal forma, que esta situación se relaciona con lo que plantea el Diagnóstico Educativo Comunitario DEC (2003): "El calendario escolar impide el acceso y permanencia de la niñez en la escuela, porque no toma en cuenta las épocas de siembra, cosecha. El sistema escolar no funciona con un calendario adecuado a los ciclos de producción y a las necesidades de las comunidades. Para los padres de familia, en la mayoría, ante esta situación ellos dan otras explicaciones, que tienen que ver con garantizar la seguridad alimenticia, de esa manera las niñas y niños asistan a clase bien alimentados y así van aprender mejor."

En la reunión del grupo focal de las madres y padres de familia dijeron que la alimentación para la niñez es bien baja, algunos se van a clase sin comer nada, no es porque no se trabaje, sino que a veces la producción es mala, y entonces los próximos años lo que hacen es duplicar la cantidad de siembra: necesitando la mano de obra de los niños. Sobre el mismo caso un padre de familia dijo: "Si le damos a los niños solamente el banano o yuca, los niños se enferman, pero si le damos a los niños arroz, fríjol, y otra cosa, entonces los niños ya se sienten bien". 
Una madre de familia planteó que la actividad agrícola afectaba la asistencia de la niñez, pero es porque hay muchas madres solteras y para hacer los trabajos necesitan la mano de obra de sus hijas e hijos y como no cuentan con recursos se ven obligados a llevarlos para que les ayuden a sembrar o por lo menos a traer un galón de agua en los trabajos duros, esto se da principalmente en los tiempos de siembra, la deshierba, la cosecha de los granos. Aunque en su totalidad coincidieron que la ausencia de la niñez les causa atrasos en el aprendizaje: porque los contenidos desarrollados los desconocen y cuando realizan pruebas o exámenes de evaluación no rinden, o se aplazan. Pero, también decía el director del centro, que existen padres de familia que cuando sus hijos están matriculados en la escuela aunque se vean en la necesidad de que los apoyen durante la siembra no los hacen perder los días de clase, los apoyan para que sigan con sus estudios, porque sueñan con ver los frutos de sus esfuerzos.

De igual manera una señora Viuda, dijo: "Soy viuda, tengo muchas responsabilidades a mi cargo, así como necesidades; pero, nunca voy a ser que mis hijos pierdan sus clases sólo por llevármelos a un trabajo, más bien busco la manera para que mi hijo se dedique más al estudio para que vaya aprendiendo ya que no tiene padre y tengo que luchar por ellos". La mayoría de los entrevistados plantearon que por la necesidad se ven obligados a llevar a sus hijos al trabajo por ser pobres, aunque algunos diferían ya que decían que aunque sean pobres nunca dejarían que sus hijas pierdan sus clases, más bien les brindarían todo el apoyo para que estudien.

Enfermedades: Durante el recorrido que se realizó, se pudo constatar que existen muchos problemas de enfermedad en la comunidad; pero, en la mayoría son los niños los afectados. Los padres y madres de familia, decían que hay muchas enfermedades que afectan más a la niñez, por lo que se ven obligados a retirarse de la escuela y pierden el año escolar. Manifestaron que las enfermedades más comunes en la comunidad son: dolor de cabeza, mareos o nauseas, fiebre y diarrea, pero que en los últimos tiempos se está generando más el problema del dolor de cabeza, mareos y convulsiones en las muchachas jóvenes el cual ellos identifican como grisi siknis, situación que tiene muy preocupada a la comunidad.

Este fenómeno de las enfermedades que se está produciendo en la comunidad, tiene mucha relación con lo que plantea el informe presentado por El Instituto del Desarrollo Pedagógico (IDEP) de la Secretaría de Educación de Bogotá, manifestando su preocupación por el crecimiento en las dificultades de aprendizaje como consecuencia de las enfermedades que afectan a los educandos, por lo que se requiere de la utilización de pedagogías flexibles y acorde con las circunstancias de la salud de los niños. http:// www.universia.net.co/noticias/noticia-del-dia/la-dificultad-de-aprender.htm/. 
Ante esta situación de enfermedades en los niños, el docente debe buscar nuevas estrategias de enseñanza en las aulas de clase, porque su estado de ánimo y mental se diferencia con los otros niños del aula de clase y por eso se debe atender de manera diferenciada a esta niñez. Un padre de familia manifestó que los niños pierden su clase por problemas de enfermedad, los afecta el factor socioeconómico porque después del huracán Félix, hay problemas de alimento, y los niños se enferman por no alimentarse bien por lo que no pueden asimilar bien la asignatura y el aprendizaje es deficiente. (Grupo focal padres y madres, 2008). En el aula de clase, encontramos niños con fiebre y otros tipos de enfermedades y estaban aislados del grupo, por lo que consideramos que se les debería otorgar tratamiento, estableciendo así una estrecha relación con lo que plantea el informe de la Secretaria de Educación de Colombia, al referirse que las enfermedades más comunes detectadas en las zonas rurales son dolor de cabeza, fiebre, vómitos, diarrea, ocasionando la desnutrición en los niños, así como deficiencias motoras en la escritura y aprendizaje de las lenguas.

Práctica del español en diversos contextos: La comunidad de "Santo Tomás", se encuentra cerca de la comunidad mestiza "San Juan Bodega", así que la mayoría de la población adulta son trilingües al comunicarse en idiomas: mayangna, miskitu y español, esta situación se da por los constantes contactos que tienen los adultos y jóvenes con los miembros de esta comunidad; empero, esta situación diverge en la niñez, ya que estos solamente socializan entre los misma niñez de la comunidad, por lo tanto esto hace difícil que puedan practicar el español en los diversos espacios de la comunidad.

Esta situación se relaciona con lo que refiere Venezia (2003) que en muchas poblaciones indígenas en las cuales no se tiene un contacto constante con poblaciones de habla hispana, los niños y niñas llegan a la escuela con el dominio correspondiente a su edad de habilidades comprensivas y expresivas de su lengua materna a nivel oral, pero con un manejo incipiente o nulo de esas habilidades en el español, considerado su segunda lengua.

Durante las observaciones se constató, en el contexto de la escuela durante el receso: las niñas y niños solamente usan el mayangna y el miskitu; sin embargo, durante el desarrollo de las clases solamente la maestra hace uso del español fuera de la escuela. Asimismo se comprobó que los días domingo durante la escuela dominical, la niñez 
memoriza textos bíblicos en español, pero no de manera fluida. Hay así una cierta relación con lo que dice De la Torre (1997) en relación a que la socialización del español está basado en diversos intereses de la familia y de la misma sociedad. La adquisición del español es considerado como medio fundamental para alcanzar un mayor nivel de educación y mejores oportunidades de trabajo en la ciudad, aunque para los padres de familia en la mayoría sus expectativas están centradas en que sus hijos aprendan el español; pero, si la situación sociolingüística en la comunidad es adversa, difícilmente los niños podrán aprender a hablar la lengua dentro de la comunidad.

\section{Conclusiones}

Existen diversos factores pedagógicos tales como falta de capacitación, carencia de materiales educativos: textos, programas y guías metodológicas que dificultan el proceso de enseñanza y aprendizaje del español como segunda lengua ( L2). La falta de aplicación de una metodología apropiada y funcional para la enseñanza de una segunda lengua, es otro de los factores que limita el aprendizaje del español en los niños mayangnas del segundo grado. La ausencia de acompañamiento y asesoría pedagógica a la maestra de segundo grado, por parte de los técnicos metodólogos del PEBI y autoridades educativas, dificulta un buen desarrollo del proceso de enseñanza aprendizaje y por ende el aprender a hablar el español como segunda lengua (L2).

La existencia de consonante en la lengua mayangna, que son muy particulares, como $\mathrm{f} / \mathrm{p} / \mathrm{ng} / \mathrm{mh} / \mathrm{rh} / \mathrm{lh}$ y que se diferencian del español, a la hora de la pronunciación de los sonidos de la L1 a la L2, suele confundir a la niñez a la hora de practicar el español, de igual manera en su escritura. La no aplicación de orden en el desarrollo de las habilidades lingüísticas, básico para la comunicación y la lecto-escritura (Escuchar, hablar, leer y escribir) limita que los niños aprendan el español en su forma oral e inclusive escrita. El ciclo de producción de la comunidad, afecta de manera negativa la participación de las niñas y niños en la escuela, porque los períodos de siembra y cosecha no coinciden con las vacaciones de la niñez y esto hace que el aprendizaje de español, no sea sistemático, sino descontinúo y de hecho no se aprenda a hablar. Las enfermedades han sido un factor que influye de manera negativa en la niñez en cuanto la asistencia a la escuela, ya que hace que pierdan muchos días de clase dificultando el aprendizaje del español como segunda lengua (L2). 


\section{Literatura citada:}

Chiriboga, Bolívar (1993). Didáctica del español como segunda lengua. Quito Ecuador P.EBI (MEC-GTZ) \& Abya-Yala.

De la Torre, Luís (1998). Experiencias de Educación Intercultural Bilingüe en Latinoamérica. Ediciones Abya Yala. Quito Ecuador.

Freeland Jane (2003). Lengua. 1era. Edición, Managua.Terra Nuova.

Kuper Wolfgan (1993). Investigación Pedagógica Intercultural Bilingüe. PEBI (,EC-GTZ) Ediciones Abya Yala. Quito Ecuador.

Moya Ruth (1996). Desde el aula Bilingüe. Proyecto de educación Maya, Xelajuj Noj Quetzaltenango, Guatemala.

Maria E. Fernández (2007).Universidad Pedagógica Experimental.

Muñoz C. Héctor (2000). Miradas y voces en torno a la educación bilingüe indígena. Ciudad de México.

MED/CETT. Documento de apoyo para desarrollar la compresión lectora. P.6.

URACCAN - IPILC (2004). Diagnóstico Educativo Comunitario en la Costa Caribe Nicaragüense. Managua, Nicaragua.

MINED (2007) Plan nacional de educación. Managua, Nicaragua.

Godoy Alfaro, Azucena (2008). MINED, Rosita, R.A.A.N. Entrevista 23 de Mayo 2008.

Venecia Mauceri, Paolo (2003). Educación Intercultural y plurilingüe. 1era. Edición Managua.

http://www.universia.net.co/noticias/noticia-del-dia/la-dificultad-de-aprender. $\underline{\mathrm{htm} /}$ 